\title{
Editorial: ACM/Springer Mobile Networks \& Applications - Special Issue on Mobile Service Computing and Applications
}

\author{
Honghao Gao ${ }^{1}$ Jianwei Yin ${ }^{2} \cdot$ Gongzhu $\mathrm{Hu}^{3}$ \\ Published online: 26 June 2019 \\ (C) Springer Science+Business Media, LLC, part of Springer Nature 2019
}

\section{Editorial:}

Service computing covers the science and technology of bridging the gap between business and IT services, and has attracted increasing attention from both industry and academia. Services are defined as software artefacts that are autonomous, self-described, reusable, and highly portable. They're the basic units for building rapid, low-cost, secure, and reliable applications. Thus, the service computing paradigm saves on development costs that would otherwise be spent creating new software components for each new business process.

Due to the rapid developments in mobile devices and wireless technologies, mobile devices are play an increasing important role in our daily life. Mobile technology's huge potential brings great opportunities for traditional service computing in the mobile environment. Services are no longer limited to traditional contexts and platforms. They can be deployed on mobile devices or cloud servers and delivered over wireless networks. Mobile service computing is undoubtedly enabling us to provide and access services anytime and anywhere, which greatly facilitates our life, work, and study. However, the application of mobile service computing still faces challenges due to key limitations such as constant mobility, limited capability, restricted power, unguaranteed security, etc., which bring great challenges for both service provision and consumption. We aim to present the state-of-the-art research on mobile service computing, as well as to provide a forum for

Honghao Gao

gaohonghao@shu.edu.cn

Jianwei Yin

zjuyjw@cs.zju.edu.cn

Gongzhu $\mathrm{Hu}$

hu1g@cmich.edu

1 Shanghai University, Shanghai, China

2 Zhejiang University, Hangzhou, China

3 Central Michigan University, Mount Pleasant, MI, USA

experts to disseminate their recent advances and views on future perspectives in the field.

This special issue features six selected papers with high quality. The first article, titled "An Approach to Alleviate the Sparsity Problem of Hybrid Collaborative Filtering Based Recommendations: The Product-Attribute Perspective from User Reviews", proposed a sparsity alleviation recommendation approach to solve the sparsity problem by addressing the zero values. Based on the Multiplication Convergence Rule and Constraint Condition, the algorithm replaces zero values through equations. The sparsity problem of the Product Attribute Model was alleviated in view of the accuracy of matrix factorization. Moreover, a hybrid collaborative formula that incorporates product attribute information is proposed to generate better recommendation results.

The second article, titled "QoS Prediction for Service Recommendation with Deep Feature Learning in Edge Computing Environment", presented Joint CNN-MF mode, with a matrix factorization (MF) model using deep features learning. The MF model integrates a convolutional neural network $(\mathrm{CNN})$ for improving the recommendation accuracy of service recommendation, where $\mathrm{CNN}$ is adopted to learn the neighbors features, forms a feature matrix and infers the features of the target user or target service.

In the next article with the title "A New Deep LearningBased Handwritten Character Recognition System on Mobile Computing Devices", the authors designed an image processing module for a mobile device based on the characteristics of a CNN. And they described how to use the mobile to collect data, process the data, and construct the data set. Moreover, considering the computing environment and data characteristics of mobile devices, they proposed a lightweight network structure for optical character recognition (OCR) on specific data sets.

To reduce communication barriers and enhance the threedimensional (3D) feel of immersive interactions is one of the most important topics for mobile applications. The fourth article, titled "An Augmented Reality-Based Method for 
Remote Collaborative Real-Time Assistance: from a System Perspectives", proposed a multiplayer real-time video communication framework with WebRTC, which enables remote experts to observe a first-hand view of an operator's site. Also, a shared cross-platform virtual whiteboard based on Canvas, WebSocket and Node.js is developed to provide remote experts with visual assistance, such as drawings or text, and adjust the position of the whiteboard for seamless integration with video. Furthermore, the virtual assistance information was displayed on the screen of AR holographic glasses to enhance the assistance capability of the platform.

The fifth article, titled "Deploying Data-intensive Applications with Multiple Services Components on Edge", proposed a Data-intensive Service Edge deployment scheme based on Genetic Algorithm (DSEGA). A data-intensive edge service composition and an edge server model was generated based on a graph theory algorithm, then five algorithms of Genetic Algorithm (GA), Simulated Annealing Algorithm (SA), Ant Colony Algorithm (ACO), Optimized Ant Colony Algorithm (ACO v) and Hill Climbing (HC) were respectively used to obtain an optimal deployment scheme, so that the response time of the data-intensive edge service deployment reaches a minimum under storage constraints and load balancing conditions.

The last article, titled "Energy-Efficient Virtual Machine Scheduling across Cloudlets in Wireless Metropolitan Area Networks", investigated a virtual machine (VM) scheduling method, to balance the implantation time and the energy consumption to cope with the above challenge. Specifically, a collection of available migration polices are obtained through heuristically searching of destination cloudlets for the running computing tasks. Then, simple additive weighting (SAW) and multiple criteria decision making (MCDM) techniques are leveraged to select the optimal VM scheduling strategy across cloudlets in WMAN.

Acknowledgements The guest editors are thankful to our reviewers for their effort in reviewing the manuscripts. We also thank the Edit-in-Chief, Dr. Imrich Chlamtac for his supportive guidance during the entire process.

Publisher's Note Springer Nature remains neutral with regard to jurisdictional claims in published maps and institutional affiliations.

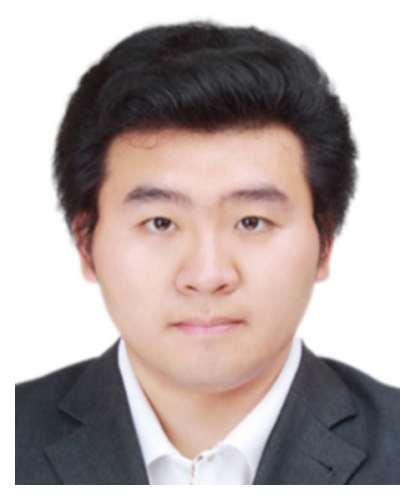

Honghao Gao received the Ph.D. degree in Computer Science and started his academic career at Shanghai University in 2012. He is an IET Fellow, BCS Fellow, EAI Fellow, IEEE Senior Member, CCF Senior Member, and CAAI Senior Member. Dr. Gao is currently a Distinguished Professor with the Key Laboratory of Complex Systems Modeling and Simulation, Ministry of Education, China, and is a Research Fellow with the Software Engineering Information Technology Institute of Central Michigan University (CMU), USA. Dr. Gao's research interests include service computing, model checking-based software verification, wireless network and IoT, and sensors data application. Until now, he has approximately 40 publications in professional journals and more than 20 publications in scientific conferences including IEEE TCSS, IEEE ACCESS, IEEE TETCI, FGCS, IJDSN, WCMC, IJSEKE, Autosoft, MIS, CI, FCS, SCN, AMIS, IJCM, JIT, ICSW, SCC, SEKE, Mobiquitous, TASE, CollaborateCom, and SNPD, obtained 13 patent applications and registered 8 software copyrights in China involving intelligent vehicle, searching engine, cloud rendering, service composition verification and testing, and IoT application under IPv4/IPv6. He has been served as Lead Guest Editor for ACM TOMM, IEEE TITS, IEEE ACCESS, Wiley ETT, ComSIS, CI, IJDSN, MONET, WINE, JIT, IJSEKE, JOEUC, JMIHI, CAI, and organized more than 20 International Conferences and Workshops, such as CollaborateCom, ChinaCom, TRIDENTCOM, Broadnest, MobiCASE, FMSC, DISA, SSIA, IEEE/ACIS ICIS and ACTI.

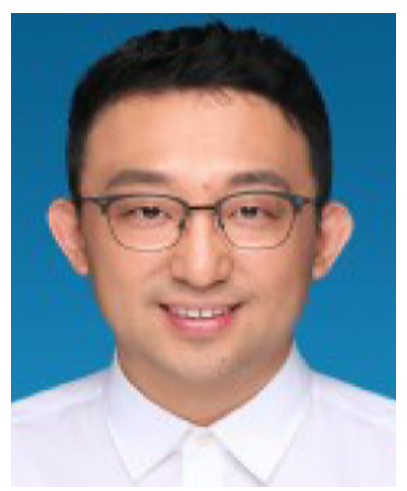

Jianwei Yin received the Ph.D. degree from Zhejiang University, Hangzhou, China, in 2001. He is currently a Full Professor with the College of Computer Science and Technology, Zhejiang University. His research interests include service computing, middle-ware, semantic Web, cloud computing, workload analysis, and performance prediction. He has published over 120 research papers in major peer-reviewed international journals and conference proceedings in the above areas. 


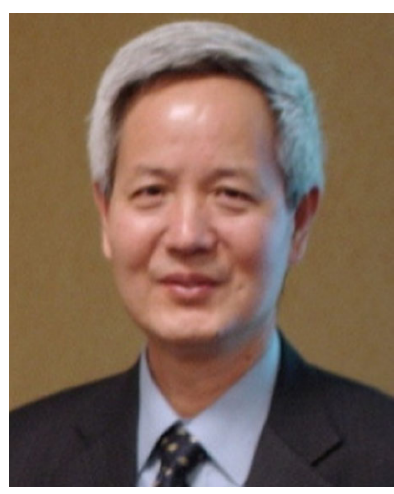

Gongzhu Hu received BS in numeric analysis from Tsinghua University, China, MS in computer science from the University of Wisconsin-Madison, and $\mathrm{PhD}$ in computer science from Michigan State University. He joined the Computer Science Department at Central Michigan University in 1987 and is currently a professor of the department. He was the department chair from 1994 to 2007.

His research interests include data mining, databases, distributed systems, and formal methods for software components. He has published over 160 papers in refereed journals and conference proceedings. Dr. Hu has served as the conference chair or program chair of many international conferences, and a member of the editorial board of the several international journals. He is a member of ACIS, ACM, a senior member of IEEE, and a senior member of ISCA. Dr. Hu served as the President of ACIS from 2010 to 2017, and a member of Board of Directors of ACIS and ISCA. 